\title{
Transient Global Amnesia: an emotional trigger for a vascular event?
}

\section{S. Pires-Barata1, V. Neves², T. Ramires², S. Galo , C. Corzo ${ }^{1,2}$, S. Lourenço ${ }^{1,2}$, L. Rebocho² \\ Stroke Unit ${ }^{1}$, Medicine Department ${ }^{2}$, Imaging Department ${ }^{3}$; Hospital do Espírito Santo EPE Évora, Portugal}

Transient global amnesia (TGA) is described has a benign neurological condition, defined by a presence of an amnestic period (anterograde amnesia), with duration of no longer then $24 \mathrm{~h}$. The pathophysiology and etiology of this form of amnesia are not yet clear in the scientific community. The purpose of the presence study is to characterize patients with TGA.

\section{Methods}

From January 2004 to February 2018, 51 patients diagnosed with TGA (from Stroke Unit and Medicine Department) were considered eligible. A comprehensive analysis of the clinical data was performed.

\section{Results}

Mean age $63,2 \pm 8,8 y$

\section{Gender}

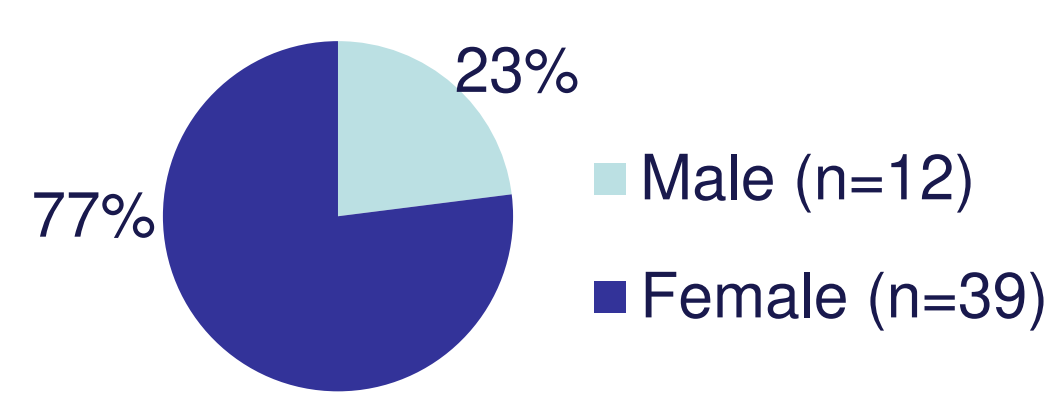

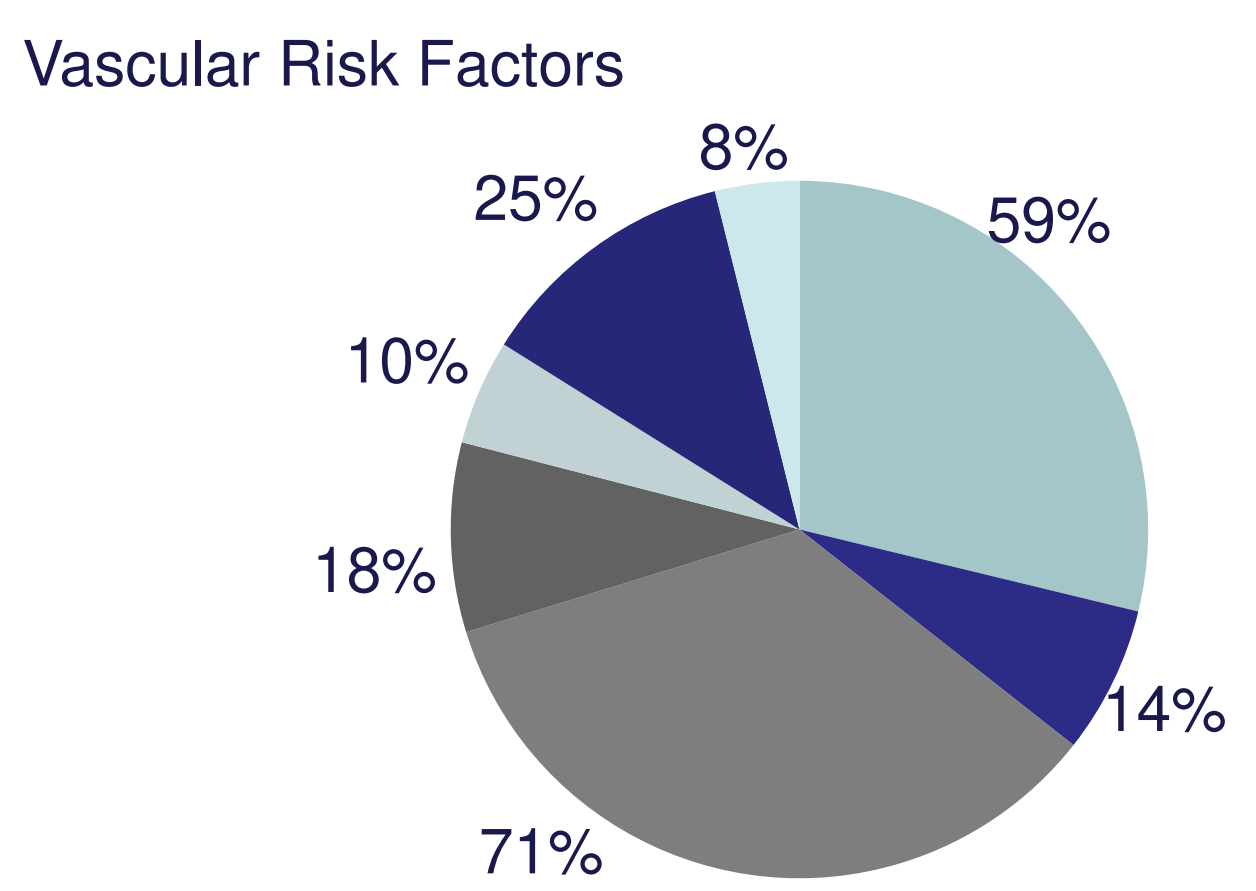

HBP

- DM

Dyslipidemia

- Obesity

Vertiginous syndrome

- Migraine

Hyperuricemia

$45,1 \%$ reported an event duration of 2-6 hours. An emotional/psychological stressful life event was reported in $64,7 \%$ of the patients (sexual intercourse, physical effort or valsalva maneuver were also reported). At admission, more than half presented a systolic $>140 \mathrm{mmHg}$.

An hyperintense signal in hippocampus (either bilateral or unilateral) was observed in $18 \%$ of the patients. During follow up period [3-6 months], MRI showed no abnormalities.

Neuropsychological Assessment: no significant previous impairment was reported (SMC; IQCODE)

* $25 \%$ presented impairment in the screening test

* $48 \%$ presented verbal initiative impairment

${ }^{*} 63 \%$ presented sleep disorders (namely secondary insomnia)

* neuropsychiatric symptoms: $39,6 \%$ depression and $56,3 \%$ anxiety
* $26 \%$ presented short term memory impairment

${ }^{*} 68 \%$ presented slow processing speed

\section{Discussion and Conclusions}

TGA was prevalence in women, with dyslipidemia and HBP as the most frequent vascular risk factors. From the follow up: no abnormalities on control MRI, the neuropsychological profile showed verbal initiative impairment, slow processing speed, sleep disorders (secondary insomnia) and anxiety symptoms.

Vascular risk factors were present, although an ischemic etiology seems to be a faraway possible explanation due to the nonexistent atherosclerotic factor. Changes in immediate memory are observed during the event, but the level of consciousness and the ability to perform other intellectual tasks are maintained. Can it be a vascular response to stress? 